\title{
Management of patients with multiple myeloma beyond the clinical-trial setting: understanding the balance between efficacy, safety and tolerability, and quality of life
}

Evangelos Terpos (10', Joseph Mikhael ${ }^{2}$, Roman Hajek $\mathbb{B}^{3}$, Ajai Chari ${ }^{4}$, Sonja Zweegman ${ }^{5}$, Hans C. Lee ${ }^{6}$, María-Victoria Mateos $\mathbb{B}^{7}$, Alessandra Larocca $\mathbb{B}^{8}$, Karthik Ramasamy ${ }^{9}$, Martin Kaiser $\mathbb{B}^{10}$, Gordon Cook ${ }^{11}$, Katja C. Weisel $\mathbb{B}^{12}$, Caitlin L. Costello ${ }^{13}$, Jennifer Elliott ${ }^{14}$, Antonio Palumbo (iD ${ }^{14}$ and Saad Z. Usmani ${ }^{15}$

\begin{abstract}
Treatment options in multiple myeloma (MM) are increasing with the introduction of complex multi-novel-agentbased regimens investigated in randomized clinical trials. However, application in the real-world setting, including feasibility of and adherence to these regimens, may be limited due to varying patient-, treatment-, and disease-related factors. Furthermore, approximately $40 \%$ of real-world MM patients do not meet the criteria for phase 3 studies on which approvals are based, resulting in a lack of representative phase 3 data for these patients. Therefore, treatment decisions must be tailored based on additional considerations beyond clinical trial efficacy and safety, such as treatment feasibility (including frequency of clinic/hospital attendance), tolerability, effects on quality of life (QoL), and impact of comorbidities. There are multiple factors of importance to real-world MM patients, including disease symptoms, treatment burden and toxicities, ability to participate in daily activities, financial burden, access to treatment and treatment centers, and convenience of treatment. All of these factors are drivers of QoL and treatment satisfaction/compliance. Importantly, given the heterogeneity of MM, individual patients may have different perspectives regarding the most relevant considerations and goals of their treatment. Patient perspectives/goals may also change as they move through their treatment course. Thus, the 'efficacy' of treatment means different things to different patients, and treatment decision-making in the context of personalized medicine must be guided by an individual's composite definition of what constitutes the best treatment choice. This review summarizes the various factors of importance and practical issues that must be considered when determining real-world treatment choices. It assesses the current instruments, methodologies, and recent initiatives for analyzing the MM patient experience. Finally, it suggests options for enhancing data collection on patients and treatments to provide a more holistic definition of the effectiveness of a regimen in the real-world setting.
\end{abstract}

Correspondence: Evangelos Terpos (eterpos@hotmail.com)

'Plasma Cell Dyscrasias Unit, Department of Clinical Therapeutics, National and Kapodistrian University of Athens, School of Medicine, Athens, Greece ${ }^{2}$ Applied Cancer Research and Drug Discovery, Translational Genomics Research Institute, City of Hope Cancer Center, Phoenix, AZ, USA Full list of author information is available at the end of the article

\section{Introduction}

Today's physicians treating multiple myeloma (MM) are faced with the challenge of individualizing treatment choices associated with the highly diverse patient populations seen across all treatment settings. Historically, median overall survival (OS) for MM patients was only $\sim 3$ years ${ }^{1}$, and there were a limited number of agents/regimens available. Now there is an increasing range of highly

\section{(c) The Author(s) 2021}

\footnotetext{
(cc) Open Access This article is licensed under a Creative Commons Attribution 4.0 International License, which permits use, sharing, adaptation, distribution and reproduction in any medium or format, as long as you give appropriate credit to the original author(s) and the source, provide a link to the Creative Commons license, and indicate if changes were made. The images or other third party material in this article are included in the article's Creative Commons license, unless indicated otherwise in a credit line to the material. If material is not included in the article's Creative Commons license and your intended use is not permitted by statutory regulation or exceeds the permitted use, you will need to obtain permission directly from the copyright holder. To view a copy of this license, visit http://creativecommons.org/licenses/by/4.0/.
} 
active treatment options available, offering novel combinations and leading to the marked improvements in patient outcomes seen in randomized clinical trials over the past 15 years $^{2}$. These changes in the MM treatment landscape make the current scenario much more complex, requiring physicians to weigh varying goals of treatment in different settings ${ }^{3-5}$. The objectives of this review are to provide a comprehensive summary of the key factors that determine treatment goals and drive treatment choices for patients-specifically, therapyrelated factors impacting patient-reported outcomes (PROs) that are additional to those related to commonly administered agents and other supportive pharmacologic interventions-and to summarize existing and emerging methodologies for capturing these drivers of treatment choices.

\section{The gap between the clinical-trial and real-world settings}

Phase 3 studies remain the 'gold standard' for obtaining regulatory approvals, based on their strong internal validity, prespecified and well-defined endpoints, and use of randomization, blinding and control arms. Favorable efficacy and benefit:risk balances have been demonstrated in clinical trials for multiple new standard-of-care regimens in recent years. However, these prospective studies have limitations in terms of external validity and generalizability. Frequently, these clinical-trial data are first reported after a median follow-up of 1-2 years, and thus the ability of patients to continue treatment beyond the initial period is unknown. The increasingly complex novel-agent-based regimens are typically associated with toxicity additional to that arising from standard backbone agents such as dexamethasone, and in the real-world setting the feasibility of and adherence to these regimens may be more difficult. The full benefit may not be derived if drugs and regimens are not: (i) tolerable enough for real-world patients and may thus impact their quality of life (QoL), including for specific patient populations such as elderly/frail patients; (ii) available to patients, e.g., due to limited mobility or travel issues/preference, or due to affordability; or (iii) in line with patients' preferences. There is a need for efficacious options that meet these criteria, and physicians require a balance of all relevant information when making treatment decisions.

Additionally, phase 3 studies may include an unrepresentative patient population. Many real-world and registry studies have concluded that approximately $40 \%$ of MM patients in the real world do not meet the criteria for inclusion in phase 3 studies on which approvals are based (Table 1$)^{6-11}$. Patients may be ineligible for a range of reasons, including poor performance status, inadequate organ function, and adverse medical history or comorbidities, meaning that they are underrepresented in phase
3 clinical trials. As documented by these studies, clinical trial ineligibility is often associated with significantly poorer outcomes compared to those reported in trialeligible patients, including shorter progression-free survival (PFS) and OS (Table 1) ${ }^{6-11}$. This leads to a lack of representative phase 3 trial data for this high proportion of real-world patients.

\section{Additional considerations in real-world patients}

In patients who are underrepresented in phase 3 clinical studies, treatment decisions must be based on additional considerations. We review multiple factors in addition to the traditional definition of efficacy that are key when considering the real-world $\mathrm{MM}$ patient experience (Fig. 1) ${ }^{12-14}$, including PROs. Those that may affect a patient's health-related QoL in the real-world setting include their disease symptoms and how they are controlled, including supportive care, adverse events (AEs) associated with therapy, and pre-existing comorbidities. Also important to patients is their ability to participate in daily activities, the support available to them, access to treatment, and-particularly for elderly/frail patientsaccess to treatment centers ${ }^{12-14}$. The level of data captured on such patient-focused outcomes is another limitation of prospective phase 3 clinical studies.

We highlight that the relative importance of these different factors, and the goals of treatment, differ between patient groups and treatment settings-suggesting that 'efficacy' does not necessarily mean the same thing to different patients and depends on the balance of all attributes of a drug or regimen. In this context, a holistic needs assessment is valuable for making treatment choices, with broad support from a multidisciplinary team in the clinic and at home, and will assist in defining efficacy/ effectiveness for each individual patient ${ }^{15,16}$. Additionally, in order to fully capture the patient's experience of their MM treatment, it is necessary to be able to analyze andwhere feasible-quantify all the relevant real-world drivers; we therefore also review the various instruments and studies developed to capture treatment impact/burden and preferences.

\section{Factors of importance to patients in the real-world setting \\ Symptom burden}

Among the hematologic malignancies, MM patients have the greatest symptom burden ${ }^{17}$. Symptoms related to the CRAB criteria (hypercalcemia, renal impairment, anemia, and bone disease) can be debilitating and may require supportive therapy such as bisphosphonates or denosumab $^{18}$. These symptoms, along with fatigue, pain, gastrointestinal symptoms ${ }^{16,19-21}$, and other common disease-related complications such as neuropathic symptoms, as well as side effects that may arise from supportive 


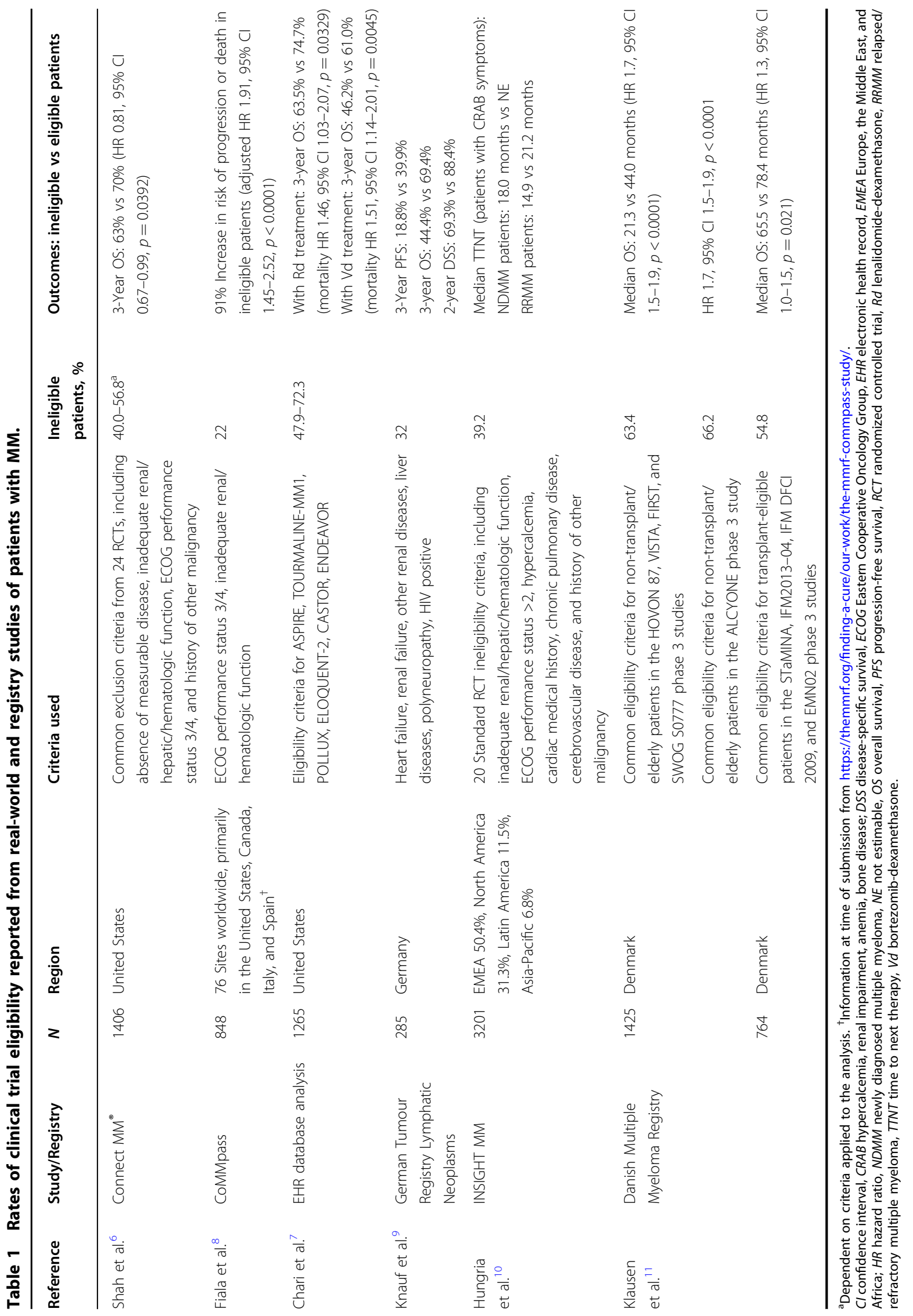




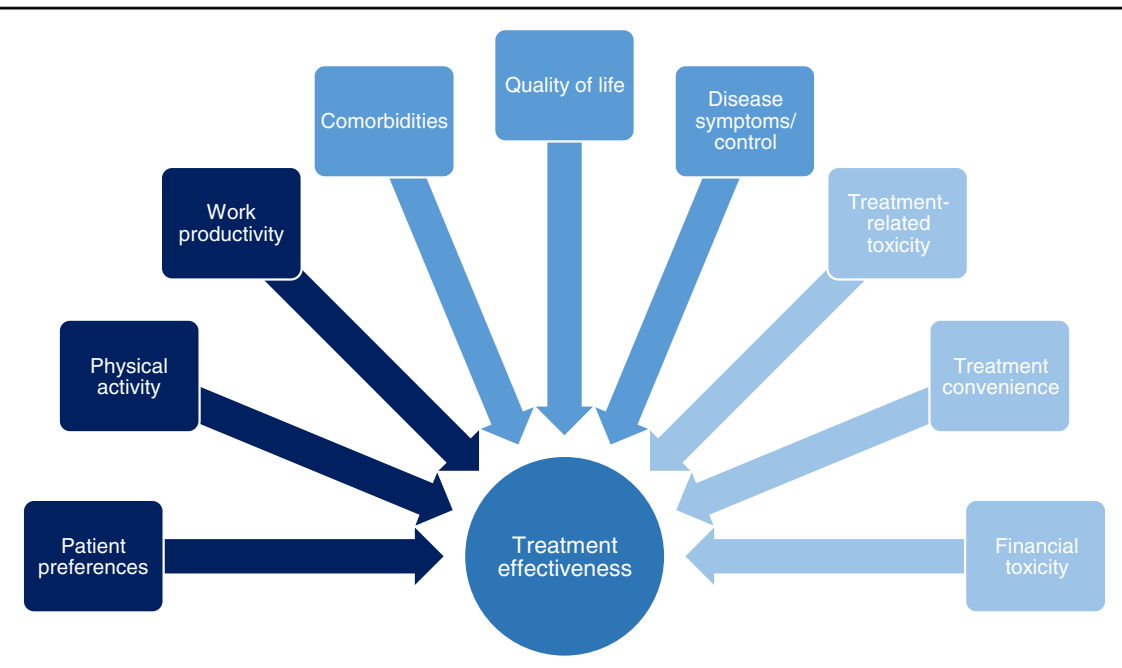

Fig. 1 Treatment factors of importance to MM patients. There are multiple factors of importance to MM patients regarding their treatment that impact on the effectiveness of that treatment in the real-world setting.

therapy, can result in MM patients having significantly impaired QoL compared to the general population $^{16,19,20,22-24}$. This highlights the need for rapid symptom control and minimal toxicity when choosing a treatment. However, in many clinical trials, patients with only biochemical progression are overrepresented.

\section{Side effects}

Real-world studies of patients' preferences have highlighted side effects of treatment as an important consideration. Various specific toxicities have been identified as being associated with specific agents, including peripheral neuropathy with bortezomib ${ }^{25}$ and thalidomide ${ }^{26}$, fatigue with lenalidomide ${ }^{27}$, cardiovascular side effects with carfilzomib ${ }^{28,29}$, gastrointestinal and hematologic side effects with ixazomib ${ }^{30,31}$, lenalidomide ${ }^{26,32}$ and panobinostat $^{33,34}$, and fluid-retention effects, bone loss, eye complications and insomnia with corticosteroids ${ }^{16}$, with associated QoL decrements having been reported due to some of these toxicities. Real-world analyses have identified the substantial role played by toxicities in treatment discontinuation in both the frontline setting $^{35,36}$ and more so in later lines ${ }^{36}$, suggesting that toxicities are more burdensome and limit the duration of treatment more substantially in the real-world setting compared with pivotal phase 3 studies. Such shortening of treatment duration due to toxicity has been shown to adversely impact outcomes ${ }^{37}$, highlighting how safety is an important component of efficacy.

\section{Daily activities}

Multiple reports have demonstrated the value to patients of being able to continue with activities of daily living and of maintaining good physical and mental well- being. Impairment of activities of daily living due to MM and its treatment or other comorbidities is associated with poorer prognosis, as demonstrated by analyses of outcomes according to frailty indices ${ }^{38,39}$, as well as patient frustration ${ }^{40}$. The ability to continue with one's daily routine and physical activities while receiving treatment is associated with fewer side effects and lower fatigue and is appreciated by patients as it improves QoL ${ }^{41-43}$.

These findings highlight the importance of gathering PROs in the context of considering the efficacy of a treatment regimen and taking into consideration the value patients place on being able to continue with their regular lives as much as possible. The associated mental health and well-being of the patient should be considered too ${ }^{14,44}$, as adverse impacts on a patient's activities and emotional functioning may curtail treatment duration and effectiveness.

\section{Financial toxicity}

Another aspect of concern to MM patients is the cost of treatment ${ }^{12,14,21,44-47}$, although the importance of this varies substantially worldwide according to healthcare system and access to drugs. Financial hardship may result from direct out-of-pocket costs arising from treatment and its side effects, depending on the healthcare system, and other indirect costs such as those involved in attending appointments (e.g., travel costs) and any compensation loss arising from impaired ability to work ${ }^{46,47}$. Studies have shown that such issues impact patients' $\mathrm{QoL}^{12,14,44}$. Thus, treatment effectiveness may also be dependent on a patient's ability to cope with the financial toxicity associated with receiving their regimen on a longterm basis. 


\section{Treatment convenience/route of administration}

Of related importance to patients is the convenience of treatment. While some patients may value the regular face-to-face contact with their treating physician/care team required with parenterally administered medications, some prefer oral medications even in the context of shorter progression-free time and/or more $\mathrm{AEs}^{48}$. This may be driven by various reasons; for example, patients may not be able to travel to infusion centers for treatment, due to limited mobility or distance from the clinic, they may wish to avoid the clinic/hospital setting due to specific circumstances, or they may want to minimize treatment burden associated with frequent hospital/clinic visits. Recent analyses have indicated patients' preference for oral treatment is based on greater convenience, less impairment of daily activities, and less impact on work/ productivity ${ }^{48-51}$. In this context, the feasibility of receiving treatment at home may be a relevant consideration, particularly in the current COVID-19 pandemic, with some studies showing domestic administration of therapy in MM patients spared the burden of repeat hospital transfers, leads to a low rate of treatment discontinuation ${ }^{52}$, and substantially improved QoL.

\section{Different patients, different perspectives: patient preferences in the real-world setting}

Patients are becoming increasingly involved in their own treatment decision-making ${ }^{53}$, and their specific preferences, including the importance they attach to each of the factors discussed in the previous section, as well as their overall treatment goals, must be considered when selecting a regimen ${ }^{54}$. As $\mathrm{MM}$ is a heterogeneous disease with a heterogeneous patient population, these preferences and goals of treatment may differ between patients, depending on multiple patient-related, diseaserelated, and treatment-related factors ${ }^{3-5,14,54,55}$. 'Efficacy' therefore means different things to different patients. Treatment decision-making in the context of personalized medicine needs to be guided by an individual's composite definition of what constitutes an effective treatment, per their preferences and treatment goals, in order to achieve the right balance between efficacy, safety, tolerability, feasibility, QoL, and treatment satisfaction ${ }^{55}$.

Within a specific patient population, drivers for treatment selection may be more granular in detail. For example, among younger MM patients, while some prioritize life expectancy/survival ${ }^{44}$, a discrete-choice experiment showed that others value preserving further treatment options, 'not always thinking of the disease', and treatment-free intervals as important characteristics of therapy, along with effectiveness ${ }^{56}$. Additionally, younger patients have been reported to rank severe or lifethreatening toxicity as a greater concern than mild or moderate chronic toxicity more frequently than older patients, associated with the need to continue working and supporting their families ${ }^{57}$. However, younger, fitter patients may opt for an intensive treatment including stem cell transplantation in order to elicit a very deep response, improve their QoL, and achieve a lengthy remission and potential functional cure ${ }^{58}$.

In contrast, among elderly/frail MM patients, preferences may differ and factors of importance may be ranked differently. Frail patients may be older and/or have more comorbidities than fitter patients, and are at a greater risk of experiencing non-hematologic toxicity and of discontinuing treatment for reasons other than progression/death ${ }^{38}$. Furthermore, frail patients are less able to receive and tolerate intensive treatment approaches intended to induce deep responses ${ }^{59}$. Thus, for some of these patients, disease control and maintaining QoL may be priorities ${ }^{2}$, with comorbidities and the challenges of polypharmacy, potential toxicities associated with treatment, and functional limitations potentially weighted more heavily when making treatment decisions ${ }^{60}$. Treatment convenience and the ability to continue with daily activities may be of substantial importance in elderly/frail patients in the context of potentially receiving longer term, less-intensive treatment regimens than younger/fit patients.

As well as differing between groups of patients, preferences and weighting of factors of importance may also differ in the same patients at different stages of their treatment course. For example, among relapsed/refractory MM patients, a primary concern is the efficacy of their treatment regimen due to the desire to get their disease back under control after experiencing relapse. While QoL in newly diagnosed MM patients may be expected to increase during/following treatment, at relapse it may be expected only to stabilize ${ }^{61}$; therefore, QoL may perhaps be weighted less heavily when choosing treatment in these patients. Nevertheless, an underlying consideration for all treatment choices is that safety and tolerability are consistent drivers for efficacy, as the longer a patient can stay on treatment, the greater the therapeutic benefit they can accrue.

\section{Measurement of PROs: analyzing real-world preferences and factors of importance}

In the context of implementing PROs, it is imperative to explore whether current QoL reporting and PRO methodologies for QoL data ${ }^{62}$ reflect all the key aspects of importance to patients and the impacts of novel treatments ${ }^{63}$. Over the past two decades a range of PRO measures (PROMs), including the European Organisation for the Research and Treatment of Cancer Quality of Life Questionnaire-Core-30 (EORTC QLQ-C30) and MMspecific (EORTC QLQ-MY20) instruments, among others 
Table 2 Patient-reported outcome measures (PROMs), physician-completed instruments, and types of studies/patientreported experience measures (PREMs) for capturing MM patients' treatment preferences and factors of importance to their treatment experience.

\begin{tabular}{|c|c|c|}
\hline Instrument/study type & Factors addressed & Scope/methodology \\
\hline EORTC-QLQ-C30 ${ }^{68}$ & $\begin{array}{l}\text { QoL, including physical and emotional } \\
\text { functioning, symptoms, and toxicity } \\
\text { Financial toxicity }\end{array}$ & $\begin{array}{l}\text { 30-ltem instrument: } \\
\text { Global Health Status/QoL scale } \\
5 \text { Functional scales (physical, role, cognitive, emotional, social) } \\
3 \text { Symptom scales (fatigue, nausea and vomiting, pain) } \\
6 \text { Single-item scales (appetite loss, diarrhea, dyspnea, constipation, } \\
\text { insomnia, financial impact) }\end{array}$ \\
\hline EORTC-QLQ-MY2030 & $\begin{array}{l}\text { QoL, including physical and emotional } \\
\text { functioning, symptoms, and toxicity }\end{array}$ & $\begin{array}{l}\text { 20-Item instrument: } \\
4 \text { Domains (disease symptoms, side effects of treatment, body image, } \\
\text { future perspectives) }\end{array}$ \\
\hline FACT-MM ${ }^{103}$ & $\begin{array}{l}\text { Symptoms } \\
\text { Physical activities } \\
\text { Emotional well-being }\end{array}$ & $\begin{array}{l}\text { 14-Item instrument covering symptoms, impact on physical activities, } \\
\text { and emotional well-being selected by expert clinicians and MM } \\
\text { patients }\end{array}$ \\
\hline MyPOS $^{19}$ & $\begin{array}{l}\text { Symptoms } \\
\text { Physical activities } \\
\text { Emotional well-being } \\
\text { Supportive care } \\
\text { Financial toxicity }\end{array}$ & $\begin{array}{l}\text { 19-Question instrument covering } 11 \text { specific symptoms and questions } \\
\text { regarding physical activities, emotional well-being, and } \\
\text { supportive care }\end{array}$ \\
\hline $\begin{array}{l}\mathrm{EQ}-5 \mathrm{D}-3 \mathrm{~L} / 5 \mathrm{~L}^{19} \\
\text { Time-trade-off utility measure }\end{array}$ & $\begin{array}{l}\text { Physical activities } \\
\text { Emotional well-being } \\
\text { QoL }\end{array}$ & $\begin{array}{l}\text { 5-Item instrument (mobility, self-care, usual activities, pain/discomfort, } \\
\text { anxiety/depression), plus visual analog scale for Global Health Status }\end{array}$ \\
\hline Katz ADL scale $e^{38}$ & Physical functioning / activities & $\begin{array}{l}\text { 6-Item instrument capturing whether a patient can/cannot perform } \\
\text { key self-care activities }\end{array}$ \\
\hline Lawton IADL scale ${ }^{38}$ & Physical functioning / activities & $\begin{array}{l}\text { 8-Item instrument capturing whether a patient can/cannot perform } \\
\text { key routine activities }\end{array}$ \\
\hline Brief Pain Inventory & $\begin{array}{l}\text { Pain and impact on physical functioning / } \\
\text { activities and emotional functioning / QoL }\end{array}$ & $\begin{array}{l}\text { 9-Question (short form) or 32-question (long form) instrument } \\
\text { evaluating current, overall, and worst pain experienced, and impact on } \\
\text { activities, mood, and QoL }\end{array}$ \\
\hline Stated preference study ${ }^{57}$ & Patient preference & $\begin{array}{l}\text { Multi-criteria decision analysis study; patients state preferences for a } \\
\text { range of specific attributes of treatment }\end{array}$ \\
\hline $\begin{array}{l}\text { Direct preference } \\
\text { assessment }^{5,64}\end{array}$ & Weighting of factors of importance & $\begin{array}{l}\text { Multi-attribute / multi-characteristic analysis study; patients rate } \\
\text { importance of each attribute / characteristic }\end{array}$ \\
\hline $\begin{array}{l}\text { Discrete-choice } \\
\text { experiment }{ }^{48,56,64}\end{array}$ & Patient preference & $\begin{array}{l}\text { Multi-attribute / multi-characteristic analysis study; patients indicate } \\
\text { treatment preferences between discrete sets of pairs of characteristics }\end{array}$ \\
\hline Time-trade-off analysis ${ }^{65}$ & Patient preferences & $\begin{array}{l}\text { Multi-criteria valuation study; patients provide preferences for a range } \\
\text { of health states for a specific time period or dying, and state the } \\
\text { proportion of remaining time alive they would trade to be in full } \\
\text { health, without a specific state }\end{array}$ \\
\hline Value-based framework ${ }^{66,67}$ & QoL & $\begin{array}{l}\text { Evidence-based frameworks for decision-making, incorporating } \\
\text { differing levels of patient experience data }\end{array}$ \\
\hline
\end{tabular}

$A D L$ activities of daily living, EORTC European Organization for Research and Treatment of Cancer, FACT-MM Functional Assessment of Cancer-Multiple Myeloma, IADL instrumental activities of daily living, MyPOS myeloma patient outcome scale, QLQ-C30/MY20 quality of life questionnaire Core 30 module / Myeloma 20-question module.

(Table 2), have been developed and validated for MM, and a number of different types of studies have been evaluated as ways of capturing patients' treatment preferences, experience, and perspectives (Table 3$)^{12-14,44,45,48,56,57,64-67}$. Multiple studies have shown the beneficial impact of better QoL assessed using these PROMs on outcomes in MM, 
Table 3 The importance of PRO data in relation to outcomes in MM.

\begin{tabular}{|c|c|c|c|c|}
\hline Study & Regimen & Setting & $N$ & PRO data and impact on outcomes \\
\hline Ludwig et al..22 & $\mid T d-I$ & RRMM & 90 & $\begin{array}{l}\text { Significantly longer PFS (median } 10.2 \text { vs } 6.6 \text { months) and OS (median not reached vs } \\
22.9 \text { months) in patients with higher vs lower (dichotomized around median) Global Health } \\
\text { Status/QoL score on EORTC-QLQ-C30 at baseline } \\
\text { Significantly longer OS (median not reached vs } 22.9 \text { months) in patients with higher vs lower } \\
\text { (dichotomized around median) physical functioning score at baseline }\end{array}$ \\
\hline $\begin{array}{l}\text { Strasser-Weippl } \\
\text { et al. }{ }^{71}\end{array}$ & - & NDMM & & $\begin{array}{l}\text { Psychosocial QoL scores - role functioning, emotional functioning, social functioning, } \\
\text { cognitive functioning - prognostic for OS }\end{array}$ \\
\hline Viala et al. ${ }^{72}$ & Bortezomib & RRMM & 202 & $\begin{array}{l}15 \text { PRO parameters from the EORTC QLQ-C30, EORTC QLQ-MY24, FACIT-Fatigue, and FACT) } \\
\text { GOG-NTx instruments were significant predictors for mortality on univariate analysis } \\
\text { Fatigue (OR 1.052) and physical functioning (OR 0.964) from EORTC QLQ-C30 were significant } \\
\text { predictors for mortality on multivariate analysis } \\
\text { Predictive power for mortality of clinical variables was increased by addition of PRO variables }\end{array}$ \\
\hline PROFILES registry ${ }^{68}$ & - & MM & 226 & $\begin{array}{l}\text { EORTC QLQ-C30 summary score associated (HR 0.89) with all-cause mortality in MM patients } \\
\text { Global Health Status/QoL scale (HR 0.90) and physical functioning scale (HR 0.90) also } \\
\text { associated with all-cause mortality in MM patients }\end{array}$ \\
\hline $\begin{array}{l}\text { SEER-MHOS } \\
\text { analysis }^{70}\end{array}$ & - & Elderly NDMM & 521 & $\begin{array}{l}\text { Self-reported health using the SEER-MHOS instrument dichotomized as 'high' or 'low' } \\
\text { Risk of all-cause (HR 1.32) and MM-specific (HR 1.22) mortality elevated in patients with 'low } \\
\text { vs 'high' self-reported health }\end{array}$ \\
\hline $\begin{array}{l}\text { NMSG } 4 / 90 \\
\text { analysis }^{69}\end{array}$ & MP & NDMM & 524 & $\begin{array}{l}\text { Global Health Status/QoL, physical functioning, role functioning, cognitive functioning, } \\
\text { fatigue, and pain domain scores on EORTC QLQ-C30 were statistically significant predictors of } \\
\text { survival on univariate analysis. } \\
\text { Poor physical functioning and cognitive functioning remained significant predictors of } \\
\text { survival on multivariate analysis. }\end{array}$ \\
\hline
\end{tabular}

EORTC QLQ-C30/MY20 European Organisation for the Research and Treatment of Cancer Quality of Life Questionnaire Core-30/Myeloma-specific module, FACT-BMT Functional Assessment of Cancer Therapy - Bone Marrow Transplant instrument, HR hazard ratio, IADL instrumental activities of daily living, ITd-I ixazomibthalidomide-dexamethasone plus ixazomib maintenance, KPS Karnofsky performance status, MM multiple myeloma, MP melphalan-prednisone, NDMM newly diagnosed multiple myeloma, NMSG Nordic Myeloma Study Group, OS overall survival, PFS progression-free survival, PRO patient-reported outcome, QoL quality of life, RRMM relapsed/refractory multiple myeloma, SEER-MHOS Surveillance, Epidemiology, and End Results - Medicare Health Outcomes Survey; TTP time to progression.

including OS (Table 3), highlighting that the use of PROMs in randomized clinical trials in MM provides valuable data. PROMs are particularly valuable in the context of randomization as this reduces the impact on PROs of differences in patient-/disease-related factors (although the potential for positive bias in open-label studies and due to data being gathered from those patients 'doing best' on the therapy should be acknowledged) ${ }^{22,68-72}$.

It is possible that some aspects of QoL of importance to MM patients are not being captured with the current instruments, e.g., sexual functioning ${ }^{73}$. Further, QoL data obtained using these instruments often show limited differences between treatment arms, despite significant efficacy and safety profile differences, or do not reflect specific troublesome aspects of the safety profile of a regimen, effects also reported in other malignancies ${ }^{26,61,74}$. Therefore, it should be questioned whether these and other currently available tools have sufficient sensitivity to the aspects that are of most importance to MM patients' QoL ${ }^{63}$. This may be particularly relevant if a novel agent or regimen is specifically associated with a toxicity infrequently reported with other agents, e.g., peripheral neuropathy with bortezomib or thalidomide $^{25,26}$. In such instances, discrepancies may be seen between broader and more toxicity-specific PRO tools as well as between physician-reported and patient-reported side effects ${ }^{25,75}$.

These observations have led to an increasing focus on how to implement PROs routinely and accurately in clinical practice ${ }^{76}$, and on developing a set of standardized outcome measures. Use of a standard set of PROMs would enable broader consideration of the specific factors/drivers associated with treatment effectiveness and the risk:benefit ratio for a specific therapy, thereby improving treatment decision-making for individual patients.

A number of initiatives are underway worldwide that aim to quantify the additional factors associated with treatment satisfaction in $\mathrm{MM}^{73,77}$. One such initiative, the IMPORTA project, has developed a suggested core set of outcome measures and instruments for routine collection in patients with newly diagnosed $\mathrm{MM}^{73}$, including 
conventional clinical measures of efficacy and safety plus multiple PROs covering QoL, preferences, and treatment satisfaction with a recommended collection schedule for these outcomes ${ }^{73}$.

In addition to accurately capturing all factors associated with treatment satisfaction, frequency of PRO collection is of importance with regards to capturing data in a timely manner and maximizing sensitivity to changes in QoL and patient satisfaction-if the recall window is too lengthy, treatment impacts over the time period may not be fully captured. Administration of each PRO instrument needs to be sufficiently frequent to fully capture patients' experience. PRO deterioration has been shown to precede clinical disease progression ${ }^{22,23}$, and so prospective observational studies using multiple QoL, preference, and satisfaction instruments to gather novel information may aid in routine patient management ${ }^{77,78}$.

\section{Real-world effectiveness}

The definition of efficacy is 'the ability to produce a desired or intended result'. In the context of clinical investigations, efficacy is used to define performance under ideal, controlled conditions, and primary efficacy endpoints are typically reported upfront, with secondary endpoints of safety, tolerability, and PROs providing support for the utility of novel regimens. In contrast, effectiveness refers to the performance of a regimen under real-world conditions. Consideration of PROs and their potential impact on efficacy may be more critical in the real-world setting compared to the potentially more motivating environment of clinical trial participation. Data from randomized controlled trials may not always reflect results for patients undergoing treatment in routine clinical practice, although data on some regimens indicate that duration of therapy, PFS, and/or time to next therapy are maintained between real-world non-clinicaltrial data and clinical-trial data ${ }^{79-82}$, suggesting their efficacy translates into broader effectiveness ${ }^{83}$. There are multiple potential reasons for this 'efficacy-effectiveness gap $^{80,83}$, and real-world effectiveness is dependent on multiple other endpoints beyond what is measured in clinical studies ${ }^{13}$, all of which must be considered in order to produce the desired or intended result. This suggests that, in the absence of head-to-head comparisons in clinical trials, indirect comparisons of regimens through the analysis of a single clinical trial efficacy endpoint, as is often done via network meta-analysis, may not accurately extrapolate to comparative real-world effectiveness.

Furthermore, differences between clinical trial and realworld outcomes must be considered in the context of the heterogeneous healthcare systems that exist between different countries and sometimes within individual countries. Differences in patient management and $\mathrm{MM}$ specialization between treatment centers may affect treatment outcomes, associated with factors beyond conventional efficacy and safety as determined in a clinical trial $^{80,84}$. In fact, data on real-world treatment within specialist networks ${ }^{81}$ or at specialist MM centers ${ }^{79}$ demonstrate prolonged outcomes versus, for example, claims data analyses from broader real-world practice ${ }^{85}$. One potential driver for the discrepancy in outcomes between centers participating in clinical trials and MM specialist centers, compared to broader real-world practice, may be the timing of treatment initiation at relapse. Several studies have shown that delaying treatment until symptomatic relapse occurs, compared with starting therapy at biochemical relapse, identified through regular follow-up and monitoring, may result in poorer outcomes $^{79,86,87}$. Additionally, differences in clinicians' experience with new regimens and in the availability of infrastructure for monitoring and toxicity management may also be relevant.

In the context of the above, routine collection of additional endpoints such as QoL and other PROs ${ }^{88,89}$, plus healthcare resource utilization, alongside the key efficacy and safety data collected in clinical trials, would aid in providing a complete picture of efficacy of a regimen ${ }^{90-94}$. Furthermore, as reviewed recently, there is a need to improve safety assessment and reporting in various hematologic malignancies ${ }^{95}$, with improved data collection and evaluation aiming to provide additional valuable information of relevance to the real-world effectiveness of treatments. An additional element to consider in this expanded framework of standard data collection on MM patients is the incorporation of real-world data to augment the findings of randomized controlled trials. This would further enhance the datasets available on different treatment options. More informed decisions could thus be made between treatments that include clinical trial efficacy and safety data, QoL data, patient preferences and treatment satisfaction data, economic information, and other important issues.

\section{Future perspective and recommendations}

With our increasing understanding of the differences between clinical trial patients and real-world populations, and the apparent gap between clinical trial efficacy and real-world effectiveness ${ }^{80}$, there are a number of recommendations that could enhance data collection on MM treatment regimens in the future (Fig. 2). For clinical trials there are ongoing initiatives aimed at modifying standard inclusion and exclusion criteria, for example, by removing comorbidity restrictions, which will improve the generalizability of trial findings ${ }^{96,97}$. Similar initiatives are also required for defining inclusion criteria for real-world evidence studies/analyses. Additional considerations include whether to obtain data from insurance databases or hospitals, determining the minimal requirements for 


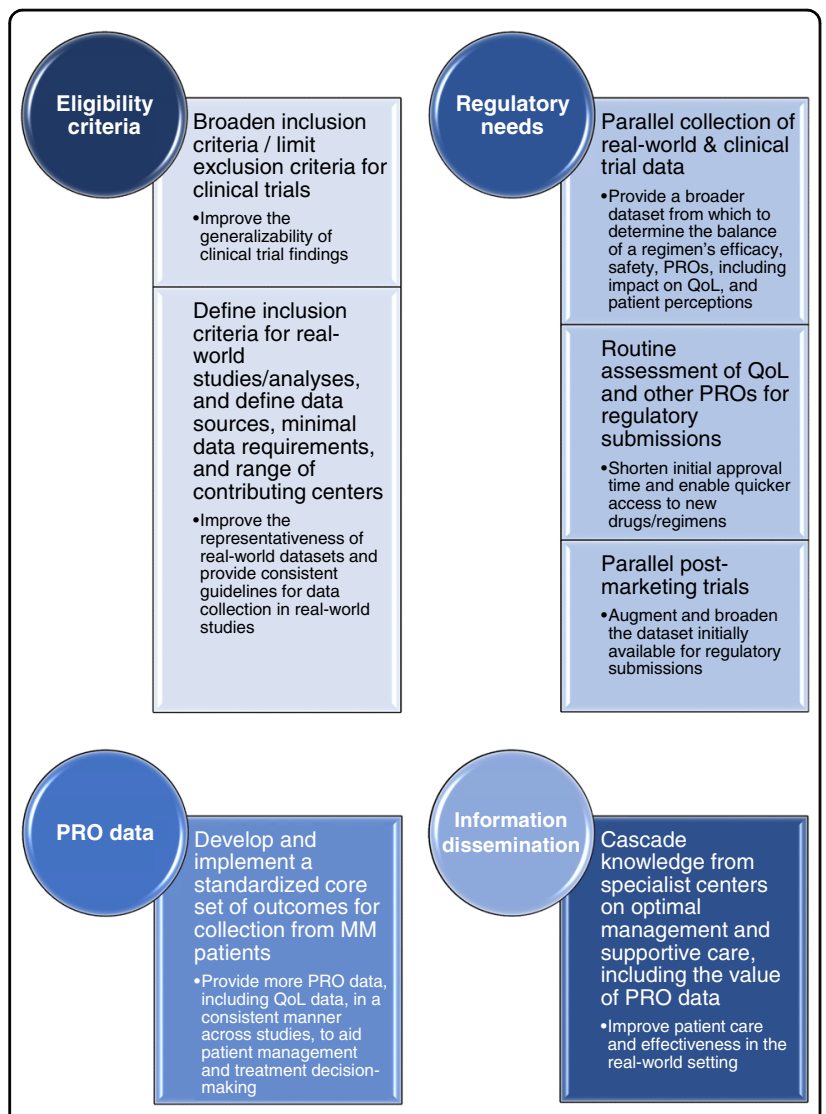

Fig. 2 Enhancing MM data collection. Recommendations for enhancing future data collection on MM treatment regimens. MM, multiple myeloma; PRO, patient-reported outcome; QoL, quality of life.

clinical data, and evaluating whether to mimic the approach of cancer registries by incorporating data from smaller, non-specialist centers or offices and including all patients seen in the contributing clinic.

From the perspective of regulatory needs, the parallel collection of real-world data and clinical-trial data should be explored in order to provide a broader dataset on which to consider approval of a regimen. Incorporating routine assessment of QoL and other PROs within a broader context of a regimen's efficacy may prove beneficial in making drugs available to patients more quickly, as many of these data are of relevance from a regulatory perspective $^{88,98,99}$. Another approach that could help speed up patient access to new regimens would be to consider parallel post-marketing trials to gather additional data to augment the dataset initially available for regulatory submissions. However, in this context it will be important to explore the current challenges with realworld data and limitations of real-world studies, and identify the need for additional or improved datasets from this setting. For example, data from registries often do not include all the data required for Health Technology Assessments, for which extra evidence is then requested.

Across both clinical trials and real-world studies, there is a need to utilize more PROMs-implementation of a standardized core set of outcome measures for collection from MM patients will help in this regard ${ }^{73}$. Similarly, data from other ongoing studies will be of interest in order to determine how best to use such PRO data on QoL, patient preferences, and patient satisfaction in the context of patient management and treatment decisionmaking $^{77}$. However, it will be important to consider the time pressures that healthcare professionals are under in their daily clinics. Completion of multiple instruments and/or analysis of multiple PROMs is likely to be too time-consuming for most, and so collection of a core set of PRO data will require a practical but comprehensive/ informative tool that does not take too much time to complete and analyze.

In light of the heterogeneous healthcare systems that can exist within individual countries, there is also a need to disseminate information more widely regarding the optimal management and supportive care of MM patients, including the value of gathering PROs, along with critical information on how to utilize different regimens and agents. Cascading this knowledge from MM specialist centers to non-specialist practices in which fewer MM patients are routinely seen will be of value. Outcomes observed in clinical trials may be impacted by suboptimal management in the real-world setting ${ }^{100}$. Thus, addressing this need may help partially close the gap seen between clinical trial efficacy and real-world effectiveness.

\section{Conclusions}

In conclusion, it is important to emphasize that selection of treatment options requires a review of the individual patients' characteristics along with careful understanding of the difference between efficacy and effectiveness, and open, honest communication with patients to appropriately define their preferences. In the modern era of MM therapy, with multiple treatment options in the frontline and relapsed settings, the isolated use of conventional clinical trial efficacy as a metric for comparisons between agents/regimens is not optimal. While efficacy data from randomized clinical trials remain the gold standard for defining the relative benefit of a regimen, safety, QoL, and other PROs are important contributors to a regimen's effectiveness in the real world. Regimens that are more tolerable and convenient for patients and have a positive impact on patients' QoL may be more likely to be administered for longer, thus enabling their effectiveness. For example, development of subcutaneous or oral instead of intravenous formulations of agents ${ }^{52,101,102}$, potentially enabling home administration $^{52,101}$, or development of novel therapeutics within a 
drug class that have lower risks of key toxicities, while preserving the efficacy, are valuable in this context.

A broader scope of additional endpoints and patientrelated factors must be considered due to their critical impact on the effectiveness of a treatment in the real world. Standardizing the collection and reporting of these endpoints and factors ${ }^{89}$, together with validation of novel instruments or composite metrics incorporating these additional considerations, will enable a broader comparison between different treatment regimens that is more meaningful to patients in the real-world setting. Elucidating how the weighting of each of the factors contributing to a regimen's effectiveness differs between groups of patients may lead to more patient-focused decision-making for tailored treatment approaches. An ultimate goal would be deriving a convenient, patientfriendly way to measure these aspects in an individual patient in a time-efficient way for physicians.

A final point to emphasize is that going forward it will be necessary to ensure that patients are more representative of the real-world MM patient population, both in clinical trials and in real-world studies/analyses. Such approaches will further support a comprehensive characterization of efficacy, safety, and PROs, including impact on QoL, associated with a treatment regimen in a representative population, thereby enabling improved treatment decision-making and personalization of therapy.

\section{Acknowledgements}

The authors acknowledge Steve Hill, Ph.D., of Ashfield MedComms, an Ashfield Health company, for professional medical writing support, which was funded by Millennium Pharmaceuticals, Inc., Cambridge, MA, USA, a wholly owned subsidiary of Takeda Pharmaceutical Company Limited, and complied with Good Publication Practice-3 (GPP3) ${ }^{104}$ guidelines, and Renda Ferrari, Ph.D. (Millennium Pharmaceuticals, Inc.) for contributing to the editorial and scientific content of the manuscript.

\footnotetext{
Author details

'Plasma Cell Dyscrasias Unit, Department of Clinical Therapeutics, National and Kapodistrian University of Athens, School of Medicine, Athens, Greece. ${ }^{2}$ Applied Cancer Research and Drug Discovery, Translational Genomics Research Institute, City of Hope Cancer Center, Phoenix, AZ, USA. ${ }^{3}$ Department of Hemato-Oncology, University Hospital Ostrava, and Faculty of Medicine, University of Ostrava, Ostrava, Czech Republic. ${ }^{4}$ Tisch Cancer Institute, Icahn School of Medicine at Mount Sinai, New York, NY, USA. ${ }^{5}$ Department of Hematology, Cancer Center Amsterdam, Amsterdam University Medical Center, VU University Amsterdam, Amsterdam, The Netherlands. 'Department of Lymphoma and Myeloma, MD Anderson Cancer Center, Houston, TX, USA. ${ }^{7}$ Department of Hematology, University Hospital of Salamanca, IBSAL, CIC, IBMCC (USAL-CSIC), Salamanca, Spain. ${ }^{8}$ Myeloma Unit, Division of Hematology, University of Torino, Azienda Ospedaliero-Universitaria Città della Salute e della Scienza di Torino, Torino, Italy. ${ }^{9}$ Department of Haematology, Oxford University Hospitals NHS Foundation Trust, RDM, Oxford University, NIHR BRC Blood Theme, Oxford, UK. ${ }^{10}$ Department of Haematology, The Royal Marsden Hospital, and Division of Molecular Pathology, The Institute of Cancer Research (ICR), London, UK. ' "Leeds Cancer Centre, Leeds Teaching Hospitals Trust, Leeds, UK. ${ }^{12}$ Department of Oncology, Hematology and Bone Marrow Transplantation with Section of Pneumology, University Medical Center Hamburg-Eppendorf, Hamburg, Germany. ${ }^{13}$ Department of Medicine, Division of Blood and Marrow Transplantation, Moores Cancer Center, University of California San Diego, La Jolla, CA, USA. ${ }^{14}$ Millennium Pharmaceuticals, Inc., a wholly owned subsidiary of Takeda Pharmaceutical Company Limited,
}

Cambridge, MA, USA. ${ }^{15}$ Department of Hematologic Oncology and Blood Disorders, Levine Cancer Institute, Charlotte, NC, USA

\section{Conflict of interest}

E.T.: Honoraria and research funding from Amgen and Sanofi; honoraria from BMS and Celgene; honoraria, travel expenses, and research funding from Genesis, Janssen, and Takeda. J.M.: Consultancy for Amgen, Celgene, GSK, Janssen, Karyopharm, Sanofi, and Takeda. R.H.: Consultancy, honoraria, membership on an entity's board of directors or advisory committees and research funding for Janssen, Amgen, Celgene and Bristol-Myers Squibb; consultancy for AbbVie; consultancy and research funding for Novartis; consultancy, honoraria and membership on an entity's Board of Directors or advisory committees for PharmaMar; consultancy, consultant or advisory relationship, honoraria, membership on an entity's board of directors or advisory committees and research funding for Takeda. A.C.: Consultancy for Janssen, Celgene, Novartis, Amgen, Bristol-Myers Squibb, Takeda, Antengene, and Secura Bio; advisory boards with Janssen, Celgene, Novartis, Amgen, Karyopharm, Sanofi, Seattle Genetics, Oncopeptides, GlaxoSmithKline, and Secura Bio; research funding from Janssen, Celgene, Novartis, Amgen, Pharmacyclics, Seattle Genetics, and Takeda. S.Z: Research funding from and advisory boards for Takeda, Celgene, and Janssen. H.C.L.: Membership on an entity's board of directors or advisory committees for Amgen, Sanofi, Celgene, Genentech, GlaxoSmithKline plc, Takeda, and Janssen; research funding from Takeda, Amgen, Janssen, Celgene, GlaxoSmithKline plc, and Daiichi Sankyo. M. V.M.: Personal fees from Takeda, Janssen, Amgen, Celgene, GSK, and Abbvie. A. L.: Advisory boards for Bristol-Myers Squibb, Celgene, Janssen, and Takeda; honoraria from Amgen, Bristol-Myers Squibb, Celgene, Janssen, and GSK. K.R.: Takeda: honoraria, research funding; Amgen: honoraria; Janssen: honoraria; Celgene: honoraria, research funding; Adaptive Biotech: Honoraria; Oncopeptides: Honoraria. M.K.: Consultancy for Amgen, Janssen, Takeda, Celgene/BMS, GSK, Karyopharm, and Abbvie; institutional research funding from Janssen and Celgene/BMS; travel support from Janssen and Takeda. G.C.: Consultancy, honoraria, research funding and speakers bureau for Janssen, Takeda and Celgene; consultancy, honoraria and speakers bureau for Sanofi and Karyopharm. K.C.W.: Advisory boards for Amgen, Bristol-Myers Squibb, Adaptive Biotech, Celgene, Janssen, Juno, Takeda, Sanofi; honoraria from Amgen, Bristol-Myers Squibb, Celgene, Janssen, and Takeda; research funding from Amgen, Celgene, Janssen, and Sanofi. C.L.C.: Honoraria and research funding for Takeda; research funding for Janssen; consultancy, honoraria and research funding for Celgene. J.E. and A.P.: Employment, Millennium Pharmaceuticals, Inc., a wholly owned subsidiary of Takeda Pharmaceutical Company Limited. S.Z.U.: Consultancy, patents \& royalties, research funding and speakers bureau for Amgen, Celgene, Janssen and Takeda; patents \& royalties and research funding for Array Biopharma and Pharmacyclics; consultancy and research funding for Bristol-Myers Squibb and Merck; patents $\&$ royalties, research funding and speakers bureau for Sanofi; consultancy for Skyline DX.

\section{Publisher's note}

Springer Nature remains neutral with regard to jurisdictional claims in published maps and institutional affiliations.

Received: 9 November 2020 Revised: 22 January 2021 Accepted: 28 January 2021

Published online: 18 February 2021

\section{References}

1. Anderson, K. C. Progress and paradigms in multiple myeloma. Clin. Cancer Res. 22, 5419-5427 (2016).

2. D'Agostino, M., Bertamini, L., Oliva, S., Boccadoro, M. \& Gay, F. Pursuing a curative approach in multiple myeloma: a review of new therapeutic strategies. Cancers (Basel) 11, 2015 (2019).

3. Davies, F. E. Is molecular remission the goal of multiple myeloma therapy? Hematology Am. Soc. Hematol. Educ. Program 2017, 205-211 (2017).

4. Mateos, M. V. \& San Miguel, J.F. Management of multiple myeloma in the newly diagnosed patient. Hematology Am. Soc. Hematol. Educ. Program 2017, 498-507 (2017). 
5. Sonneveld, P. Management of multiple myeloma in the relapsed/refractory patient. Hematology Am. Soc. Hematol. Educ. Program 2017, 508-517 (2017).

6. Shah, J. J. et al. Analysis of common eligibility criteria of randomized controlled trials in newly diagnosed multiple myeloma patients and extrapolating outcomes. Clin. Lymphoma Myeloma Leuk. 17, 575-583 e572 (2017).

7. Chari, A. et al. Randomized clinical trial representativeness and outcomes in real-world patients: comparison of 6 hallmark randomized clinical trials of relapsed/refractory multiple myeloma. Clin. Lymphoma Myeloma Leuk. 20, 8-17 e16 (2020)

8. Fiala, M. et al. The real-world characteristics and outcomes of newly diagnosed myeloma patients ineligible for clinical trials. Clin. Lymphoma Myeloma Leuk. 17, e55-e56 (2017).

9. Knauf, W. et al. Survival of non-transplant patients with multiple myeloma in routine care differs from that in clinical trials-data from the prospective German Tumour Registry Lymphatic Neoplasms. Ann. Hematol. 97, 2437-2445 (2018).

10. Hungria, V. T. M. et al. Real-world (RW) multiple myeloma (MM) patients (Pts) remain under-represented in clinical trials based on standard laboratory parameters and baseline characteristics: analysis of over 3,000 Pts from the Insight MM Global, Prospective, Observational Study. Blood 134, 1887 (2019).

11. Klausen, T. W. et al. The majority of newly diagnosed myeloma patients do not fulfill the inclusion criteria in clinical phase III trials. Leukemia 33, 546-549 (2019).

12. Baz, R. et al. Development of a conceptual model to illustrate the impact of multiple myeloma and its treatment on health-related quality of life. Support Care Cancer 23, 2789-2797 (2015).

13. Gonzalez-McQuire, S. et al. Development of an initial conceptual model of multiple myeloma to support clinical and health economics decision making. MDM Policy Pract. 4, 2381468318814253 (2019).

14. Osborne, T. R. et al. Understanding what matters most to people with multiple myeloma: a qualitative study of views on quality of life. BMC Cancer 14, 496 (2014)

15. Islam, M. S. Treat patient, not just the disease: holistic needs assessment for haematological cancer patients. Oncol. Rev. 12, 374 (2018).

16. Snowden, J. A. et al. Guidelines for screening and management of late and long-term consequences of myeloma and its treatment. Br. J. Haematol. 176, 888-907 (2017).

17. Johnsen, A. T., Tholstrup, D., Petersen, M. A., Pedersen, L. \& Groenvold, M Health related quality of life in a nationally representative sample of haematological patients. Eur. J. Haematol. 83, 139-148 (2009).

18. Terpos, E., Ntanasis-Stathopoulos, I., Gavriatopoulou, M. \& Dimopoulos, M. A. Pathogenesis of bone disease in multiple myeloma: from bench to bedside. Blood Cancer J. 8, 7 (2018).

19. Ramsenthaler, C. et al. The impact of disease-related symptoms and palliative care concerns on health-related quality of life in multiple myeloma: a multicentre study. BMC Cancer 16, 427 (2016).

20. Jordan, K. et al. Effect of general symptom level, specific adverse events, treatment patterns, and patient characteristics on health-related quality of life in patients with multiple myeloma: results of a European, multicenter cohort study. Support Care Cancer 22, 417-426 (2014).

21. Kiely, F., Cran, A., Finnerty, D. \& O'Brien, T. Self-reported quality of life and symptom burden in ambulatory patients with multiple myeloma on diseasemodifying treatment. Am. J. Hosp. Palliat. Care 34, 671-676 (2017).

22. Ludwig, $H$. et al. Quality of life in patients with relapsed/refractory multiple myeloma during ixazomib-thalidomide-dexamethasone induction and ixazomib maintenance therapy and comparison to the general population. Leuk. Lymphoma 61, 377-386 (2020).

23. Weisel, K., Ludwig, H., Rieth, A., Lebioda, A. \& Goldschmidt, H. Health-related quality of life of carfilzomib- and daratumumab-based therapies in patients with relapsed/refractory multiple myeloma, based on German benefit assessment data. Qual. Life Res. 29, 69-79 (2020).

24. Terpos, E. et al. European Myeloma Network guidelines for the management of multiple myeloma-related complications. Haematologica 100, 1254-1266 (2015).

25. Richardson, P. G. et al. Management of treatment-emergent peripheral neuropathy in multiple myeloma. Leukemia 26, 595-608 (2012).

26. Nielsen, L. K. et al. Health-related quality of life in transplant ineligible newly diagnosed multiple myeloma patients treated with either thalidomide or lenalidomide-based regimen until progression: a prospective, open-label, multicenter, randomized, phase 3 study. Haematologica 105, 1650-1659 (2019).
27. Ito, T. et al. Combined use of Ninjin'yoeito improves subjective fatigue caused by lenalidomide in patients with multiple myeloma: a retrospective study. Front. Nutr. 5, 72 (2018).

28. Chari, A. et al. Analysis of carfilzomib cardiovascular safety profile across relapsed and/or refractory multiple myeloma clinical trials. Blood Adv. 2, 1633-1644 (2018).

29. Bringhen, S. et al. Cardiovascular adverse events in modern myeloma therapy - incidence and risks. A review from the European Myeloma Network (EMN) and Italian Society of Arterial Hypertension (SIIA). Haematologica 103 1422-1432 (2018).

30. Schjesvold, F. et al. Quality of life is maintained with ixazomib maintenance in post-transplant newly diagnosed multiple myeloma: the TOURMALINE-MM3 trial. Eur. J. Haematol. 104, 443-458 (2019).

31. Kaiser, M. et al. Adverse event management in the TOURMALINE-MM3 study of post-transplant ixazomib maintenance in multiple myeloma. Ann. Hematol. 99, 1793-1804 (2020).

32. Jackson, G. H. et al. Lenalidomide maintenance versus observation for patients with newly diagnosed multiple myeloma (Myeloma XI): a multicentre, open-label, randomised, phase 3 trial. Lancet Oncol. 20, 57-73 (2019).

33. Richardson, P. G. et al. Patient-reported outcomes of multiple myeloma patients treated with panobinostat after $\geq 2$ lines of therapy based on the international phase 3, randomized, double-blind, placebo-controlled PANORAMA-1 trial. Br. J. Haematol. 181, 628-636 (2018).

34. San-Miguel, J. F. et al. Panobinostat plus bortezomib and dexamethasone versus placebo plus bortezomib and dexamethasone in patients with relapsed or relapsed and refractory multiple myeloma: a multicentre, randomised, double-blind phase 3 trial. Lancet Oncol. 15, 1195-1206 (2014).

35. Jagannath, S. et al. Real-world treatment patterns and associated progression-free survival in relapsed/refractory multiple myeloma among US community oncology practices. Expert Rev. Hematol. 9, 707-717 (2016).

36. Yong, K. et al. Multiple myeloma: patient outcomes in real-world practice. $\mathrm{Br}$. J. Haematol. 175, 252-264 (2016).

37. Bringhen, S. et al. Age and organ damage correlate with poor survival in myeloma patients: meta-analysis of 1435 individual patient data from 4 randomized trials. Haematologica 98, 980-987 (2013).

38. Palumbo, A. et al. Geriatric assessment predicts survival and toxicities in elderly myeloma patients: an International Myeloma Working Group report. Blood 125, 2068-2074 (2015).

39. Engelhardt, M. et al. A concise revised Myeloma Comorbidity Index as a valid prognostic instrument in a large cohort of 801 multiple myeloma patients. Haematologica 102, 910-921 (2017).

40. Gupta, S. et al. Assessing the effect of adherence on patient-reported outcomes and out of pocket costs among patients with multiple myeloma. Clin Lymphoma Myeloma Leuk. 18, 210-218 (2018).

41. Jackson, G. et al. Productivity losses in patients with newly diagnosed multiple myeloma following stem cell transplantation and the impact of maintenance therapy. Eur. J. Haematol. 103, 393-401 (2019).

42. Kim, S. H. et al. The importance of physical function in patients with multiple myeloma for improving quality of life. Support Care Cancer 28, 2361-2367 (2020).

43. Servadio, M. et al. Physical activity and health-related quality of life in multiple myeloma survivors: the PROFILES registry. BMJ Support Palliat. Care 10, e35 (2020).

44. Parsons, J. A. et al. Treatment preferences of patients with relapsed and refractory multiple myeloma: a qualitative study. BMC Cancer 19, 264 (2019).

45. Tariman, J. D., Berry, D. L., Cochrane, B., Doorenbos, A. \& Schepp, K. G. Physician, patient, and contextual factors affecting treatment decisions in older adults with cancer and models of decision making: a literature review. Oncol. Nurs. Forum 39, E70-E83 (2012).

46. Goodwin, J. A. et al. Personal financial effects of multiple myeloma and its treatment. Cancer Nurs. 36, 301-308 (2013).

47. Huntington, S. F. et al. Financial toxicity in insured patients with multiple myeloma: a cross-sectional pilot study. Lancet Haematol. 2, e408-e416 (2015).

48. Wilke, T. et al. Treatment of relapsed refractory multiple myeloma: which new Pl-based combination treatments do patients prefer? Patient Prefer. Adherence 12, 2387-2396 (2018).

49. Merola, D., Yong, C., Noga, S. J. \& Shermock, K. M. Costs associated with productivity loss among U.S. patients newly diagnosed with multiple myeloma receiving oral versus injectable chemotherapyJ. Manag. Care Spec. Pharm. 24, 1019-1026 (2018)

50. Rifkin, R. M. et al. Treatment satisfaction and burden of illness in patients with newly diagnosed multiple myeloma. Pharmacoecon. Open. 4, 473-483 (2019). 
51. Chari, A. et al. Patient-reported factors in treatment satisfaction in patients with relapsed/refractory multiple myeloma (RRMM). Oncologist 24, 1479-1487 (2019).

52. Cerchione, C. et al. Safety and comfort of domestic bortezomib injection in real-life experience. Support Care Cancer 26, 3111-3116 (2018).

53. Badia, $X$. et al. Patient involvement in reflective multicriteria decision analysis to assist decision making in oncology. Int. J. Technol. Assess. Health Care $\mathbf{3 5}$ 56-63 (2019).

54. Mikhael, J. et al. Treatment of multiple myeloma: ASCO and CCO Joint Clinical Practice Guideline. J. Clin. Oncol. 37, 1228-1263 (2019).

55. Fifer, S. J., Ho, K. A., Lybrand, S., Axford, L. J. \& Roach, S. Alignment of preferences in the treatment of multiple myeloma - a discrete choice experiment of patient, carer, physician, and nurse preferences. BMC Cancer 20, 546 (2020)

56. Muhlbacher, A. C., Lincke, H. J. \& Nubling, M. Evaluating patients' preferences for multiple myeloma therapy, a Discrete-Choice-Experiment. Psychosoc. Med. 5. Doc10 (2008)

57. Postmus, D. et al. Individual trade-offs between possible benefits and risks of cancer treatments: results from a stated preference study with patients with multiple myeloma. Oncologist 23, 44-51 (2018).

58. Usmani, S. Z. \& Seifter, E. Treatment approach for young, fit, newly diagnosed multiple myeloma patients. Hematology Am. Soc. Hematol. Educ. Program 2018, 97-102 (2018)

59. Larocca, A. et al. Patient-centered practice in elderly myeloma patients: an overview and consensus from the European Myeloma Network (EMN) Leukemia 32, 1697-1712 (2018).

60. Wildes, T. M. \& Anderson, K. C. Approach to the treatment of the older, unfit patient with myeloma from diagnosis to relapse: perspectives of a US hematologist and a geriatric hematologist. Hematology Am. Soc. Hematol. Educ. Program 2018, 88-96 (2018).

61. Martino, M. et al. Quality of life outcomes in multiple myeloma patients: a summary of recent clinical trials. Expert Rev. Hematol. 12, 665-684 (2019).

62. Nielsen, L. K., Abildgaard, N., Jarden, M. \& Klausen, T. W. Methodologica aspects of health-related quality of life measurement and analysis in patients with multiple myeloma. Br. J. Haematol. 185, 11-24 (2019).

63. Goswami, P., Khatib, Y. \& Salek, S. Haematological malignancy: are we measuring what is important to patients? A systematic review of quality-oflife instruments. Eur. J. Haematol. 102, 279-311 (2019).

64. Muhlbacher, A. C. \& Nubling, M. Analysis of physicians' perspectives versus patients' preferences: direct assessment and discrete choice experiments in the therapy of multiple myeloma. Eur. J. Health Econ. 12, 193-203 (2011)

65. Rowen, D. et al. Deriving a preference-based measure for cancer using the EORTC QLQ-C30. Value Health 14, 721-731 (2011).

66. Orlowski, R. Z. Letter-incorporating real-world evidence and patient value criteria into value-based frameworks for relapsed/refractory multiple myeloma. J. Manag. Care Spec. Pharm. 24, 487 (2018).

67. Djatche, L. M., Goble, J. A., Chun, G. \& Varga, S. Evaluating oncology valuebased frameworks in the U.S. marketplace and challenges in real-world application: a multiple myeloma test case. J. Manag. Care Spec. Pharm. 24 39-46 (2018).

68. Husson, O. et al. The EORTC QLQ-C30 summary score as prognostic factor for survival of patients with cancer in the "Real-World": results from the population-based PROFILES Registry. Oncologist 25, e722-e732 (2020).

69. Wisloff, F. \& Hjorth, M. Health-related quality of life assessed before and during chemotherapy predicts for survival in multiple myeloma. Nordic Myeloma Study Group. Br. J. Haematol. 97, 29-37 (1997).

70. Nabulsi, N. A et al. Self-reported health and survival in older patients diagnosed with multiple myeloma. Cancer Causes Control 31, 641-650 (2020).

71. Strasser-Weippl, K. \& Ludwig, H. Psychosocial QOL is an independent predictor of overall survival in newly diagnosed patients with multiple myeloma. Eur. J. Haematol. 81, 374-379 (2008)

72. Viala, M. et al. Patient-reported outcomes helped predict survival in multiple myeloma using partial least squares analysis. J. Clin. Epidemiol. 60, 670-679 (2007).

73. Blade, J. et al. Defining a set of standardised outcome measures for newly diagnosed patients with multiple myeloma using the Delphi consensus method: the IMPORTA project. BMJ Open. 8, e018850 (2018).

74. Schuurhuizen, $\mathrm{C}$. et al. Does severe toxicity affect global quality of life in patients with metastatic colorectal cancer during palliative systemic treatment? A systematic review. Ann. Oncol. 28, 478-486 (2017).
75. Di Maio, M. et al. Symptomatic toxicities experienced during anticancer treatment: agreement between patient and physician reporting in three randomized trials. J. Clin. Oncol. 33, 910-915 (2015)

76. Basch, E., Barbera, L., Kerrigan, C. L. \& Velikova, G. Implementation of patientreported outcomes in routine medical care. Am. Soc. Clin. Oncol. Educ. Book 38, 122-134 (2018)

77. Efficace, F. et al. A prospective observational study to assess clinical decisionmaking, prognosis, quality of life and satisfaction with care in patients with relapsed/refractory multiple myeloma: the CLARITY study protocol. Health Qual. Life Outcomes 16, 127 (2018).

78. Benaniba, L. et al. The MYRACLE protocol study: a multicentric observational prospective cohort study of patients with multiple myeloma. BMC Cancer 19 855 (2019).

79. Hajek, R. et al. Closing the efficacy and effectiveness gap: outcomes in relapsed/refractory multiple myeloma (RRMM) patients (Pts) treated with ixazomib-lenalidomide-dexamethasone $(\mathrm{IRd})$ in routine clinical practice remain comparable to the outcomes reported in the phase 3 TourmalineMM1 Study. Blood 134, 1845 (2019).

80. Richardson, P. G. et al. Interpreting clinical trial data in multiple myeloma: translating findings to the real-world setting. Blood Cancer J. 8, 109 (2018).

81. Rifkin, R. M. et al. A real-world comparative analysis of carfilzomib and other systemic multiple myeloma chemotherapies in a US community oncology setting. Ther. Adv. Hematol. 10, 2040620718816699 (2019).

82. Terpos, E. et al. Real-world effectiveness and safety of ixazomib-lenalidomidedexamethasone in relapsed/refractory multiple myeloma. Ann. Hematol. 99, 1049-1061 (2020).

83. Templeton, A. J., Booth, C. M. \& Tannock, I. F. Informing patients about expected outcomes: the efficacy-effectiveness gap. J. Clin. Oncol. 38, 1651-1654 (2020).

84. Freeman, A. T. et al. Influence of treating facility, provider volume, and patient-sharing on survival of patients with multiple myeloma. J. Natl Compr. Canc. Netw. 17, 1100-1108 (2019)

85. Chari, A. et al. Real-world outcomes and factors impacting treatment choice in relapsed and/or refractory multiple myeloma (RRMM): a comparison of VRd, KRd, and IRd. Expert Rev. Hematol. 13, 421-433 (2020).

86. Katodritou, E. et al. Real-world data on Len/Dex combination at second-line therapy of multiple myeloma: treatment at biochemical relapse is a sig nificant prognostic factor for progression-free survival. Ann. Hematol. 97, 1671-1682 (2018).

87. Lopez, A. et al. Patterns of relapse and outcome of elderly multiple myeloma patients treated as front-line therapy with novel agents combinations. Leuk. Res. Rep. 4, 64-69 (2015).

88. Bottomley, A. et al. Current state of quality of life and patient-reported outcomes research. Eur. J. Cancer 121, 55-63 (2019).

89. Coens, C. et al. International standards for the analysis of quality-of-life and patient-reported outcome endpoints in cancer randomised controlled trials: recommendations of the SISAQOL Consortium. Lancet Oncol. 21, e83-e96 (2020).

90. Ludwig, $H$. et al. Health-related quality of life in the ENDEAVOR study: carfilzomib-dexamethasone vs bortezomib-dexamethasone in relapsed/ refractory multiple myeloma. Blood Cancer J. 9, 23 (2019).

91. Tay, J. et al. Health related quality of life for multiple myeloma patients according to treatment strategy after autologous stem cell transplant: a cross-sectional study using EORTC, EQ-5D and MY-20 scales. Leuk. Lymphoma 60, 1275-1282 (2019).

92. Leleu, X. et al. Patient-reported health-related quality of life from the phase III TOURMALINE-MM1 study of ixazomib-lenalidomide-dexamethasone versus placebo-lenalidomide-dexamethasone in relapsed/refractory multiple myeloma. Am. J. Hematol. 93, 985-993 (2018).

93. Hari, P. et al. Healthcare resource utilization with ixazomib or placebo plus lenalidomide-dexamethasone in the randomized, double-blind, phase 3 TOURMALINE-MM1 study in relapsed/refractory multiple myeloma. J. Med. Econ. 21, 793-798 (2018).

94. Sparano, F., Cavo, M., Niscola, P., Caravita, T. \& Efficace, F. Patient-reported outcomes in relapsed/refractory multiple myeloma: a systematic review. Support Care Cancer 26, 2075-2090 (2018).

95. Thanarajasingam, G. et al. Beyond maximum grade: modernising the assessment and reporting of adverse events in haematological malignancies. Lancet Haematol. 5, e563-e598 (2018). 
96. Unger, J. M., Hershman, D. L., Fleury, M. E. \& Vaidya, R. Association of patient comorbid conditions with cancer clinical trial participation. JAMA Oncol. 5, 326-333 (2019).

97. Beaver, J. A., Ison, G. \& Pazdur, R. Reevaluating eligibility criteria - balancing patient protection and participation in oncology trials. N. Engl. J. Med. 376, 1504-1505 (2017).

98. Gnanasakthy, A., Barrett, A., Evans, E., D'Alessio, D. \& Romano, C. D. A review of patient-reported outcomes labeling for oncology drugs approved by the FDA and the EMA (2012-2016). Value Health 22, 203-209 (2019).

99. Basch, E. et al. Patient-reported outcomes in cancer drug development and US regulatory review: perspectives from industry, the food and drug administration, and the patient. JAMA Oncol. 1, 375-379 (2015).

100. Giri, S. et al. Underutilization of guideline-recommended supportive care among older adults with multiple myeloma in the United States. Cancer 125, 4084-4095 (2019).
101. Lassalle, A. et al. Home administration of bortezomib in multiple myeloma is cost-effective and is preferred by patients compared with hospital administration: results of a prospective single-center study. Ann. Oncol. 27, 314-318 (2016).

102. Mateos, M. V. et al. Subcutaneous versus intravenous daratumumab in patients with relapsed or refractory multiple myeloma (COLUMBA): a multicentre, open-label, non-inferiority, randomised, phase 3 trial. Lancet Haematol. 7, e370-e380 (2020).

103. Wagner, L. I. et al. Content development for the Functional Assessment of Cancer Therapy-Multiple Myeloma (FACT-MM): use of qualitative and quantitative methods for scale construction. J. Pain Symptom Manage. 43 1094-1104 (2012).

104. Battisti, W.P. et al. Good publication practice for communicating companysponsored medical research: GPP3. Ann. Intern. Med. 163, 461-464 (2015). 